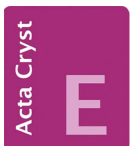

CRYSTALLOGRAPHIC COMMUNICATIONS

ISSN 2056-9890

Received 19 April 2016

Accepted 25 April 2016

Edited by M. Zeller, Purdue University, USA

Keywords: crystal structure; zinc; benzene1,3,5-tricarboxylic acid; metal-organic framework.

CCDC reference: 1476509

Supporting information: this article has supporting information at journals.iucr.org/e

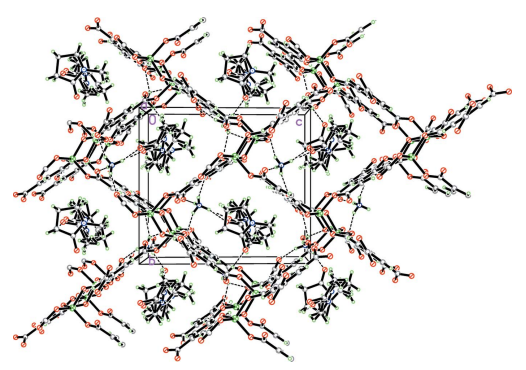

OPEN $\odot$ ACCESS

\section{Crystal structure of poly[bis(ammonium) [bis $\left(\mu_{4}-\right.$ benzene-1,3,5-tricarboxylato)dizincate] 1-methyl- pyrrolidin-2-one disolvate]}

\author{
Carlos Ordonez, ${ }^{\mathrm{a}}$ Marina S. Fonari, ${ }^{\mathrm{b} *}{\text { Qiang } \mathrm{Wei}^{\mathrm{a}} \text { and Tatiana V. Timofeeva }}^{\mathrm{a}}$ \\ ${ }^{\mathbf{a}}$ Department of Biology \& Chemistry, New Mexico Highlands University, Las Vegas, NM 87701, USA, and ${ }^{\mathbf{b}}$ Institute of \\ Applied Physics, Academy of Sciences of Moldova, Academy str. 5, MD2028 Chisinau, Republic of Moldova. \\ *Correspondence e-mail: fonari.xray@gmail.com
}

The title three-dimensional metal-organic framework (MOF) compound, $\left\{\left(\mathrm{NH}_{4}\right)_{2}\left[\mathrm{Zn}_{2}\left(\mathrm{C}_{9} \mathrm{H}_{3} \mathrm{O}_{6}\right)_{2}\right] \cdot 2 \mathrm{C}_{5} \mathrm{H}_{9} \mathrm{NO}\right\}_{n}$, features an anionic framework constructed from $\mathrm{Zn}^{2+}$ cations and benzene-1,3,5-tricarboxylate (BTC) organic anions. Charge balance is achieved by outer sphere ammonium cations formed by degradation of di- $n$-butylamine in the solvothermal synthesis of the compound. Binuclear $\left\{\mathrm{Zn}_{2}(\mathrm{COO})_{2}\right\}$ entities act as the framework's secondary building units. Each $\mathrm{Zn}^{\mathrm{II}}$ atom has a tetrahedral coordination environment with an $\mathrm{O}_{4}$ set of donor atoms. The three-dimensional framework adopts a rutile-type topology and channels are filled in an alternating fashion with ordered and disordered 1-methylpyrrolidin-2-one solvent molecules and ammonium cations. The latter are held in the channels via four $\mathrm{N}-\mathrm{H} \cdots \mathrm{O}$ hydrogen bonds, including three with the benzene-1,3,5-tricarboxylate ligands of the anionic framework and one with a 1-methylpyrrolidin-2-one solvent molecule.

\section{Chemical context}

1,3,5-Benzenetricarboxylic acid $\left(\mathrm{H}_{3} \mathrm{BTC}\right)$ has proved its efficacy as a versatile and powerful ligand for the construction of metal-organic frameworks (MOFs). Its three carboxylate groups and benzene ring can act as short and long bridges between metal ions, leading to three-dimensional assemblies with a large structural diversity (Eddaoudi et al., 2001; Almeida Paz \& Klinowski, 2004; Liu et al., 2007). Since 1997 (Yaghi et al., 1997), the coordination chemistry of zinc ions and BTC ligands has represented one of the most extensively explored systems in efforts to synthesize new porous materials. The various aspects of the $\mathrm{Zn}-\mathrm{BTC}$ system continue to being investigated, and diverse MOF structures have been reported. The published results reveal that the variation of starting compositions, solvents and templates as well as reaction conditions are significant and can result in the formation of completely different metal-organic framework compounds. A base is needed for deprotonation of $\mathrm{H}_{3} \mathrm{BTC}$ so that it can make use of its full coordination capacity. This base should have a low affinity for binding to metal ions to avoid competition with BTC, especially if the aim is the synthesis of porous materials. A wide range of different solvent systems and reaction conditions have been used in the construction of new coordination networks, including the use of ionothermal techniques (Xu et al., 2007), and conducting reactions in the presence of different surfactants as reaction media (Gao et al., 2014). 
In our recent work (Ordonez et al., 2014), we reported 13 different Zn-BTC coordination networks that were formed as a result of the use of different cations as framework templates. Generally, only one type of secondary building unit (SBU) is observed in one compound; however, data from our and other groups (Ordonez et al., 2014; Xie, 2013; Hao et al., 2012) have shown the possibility of different SBUs in a single selfassembled system which can, in turn, result in distinct frameworks and topologies. In some cases, hydrothermal reaction conditions lead to decomposition of solvents or bases (Burrows et al., 2005), and fixation of the decomposition products in the systems can result in unexpected guests such as ammonium cations (Ordonez et al., 2014). Herein we report the structure of a new three-dimensional Zn-BTC MOF obtained serendipitously by reaction of the $\mathrm{H}_{3} \mathrm{BTC}$ ligand with zinc nitrate hexahydrate using 1-methylpyrrolidin-2-one (NMP) as a solvent and di-( $n$-butyl)amine as a base and a framework template. The main product of the reaction was the $\{\mathrm{Zn}-\mathrm{BTC}\}\left\{n-\mathrm{Bu}_{2} \mathrm{NH}_{2}\right\} \mathrm{MOF}$, but a few single crystals of title compound were found as a byproduct.

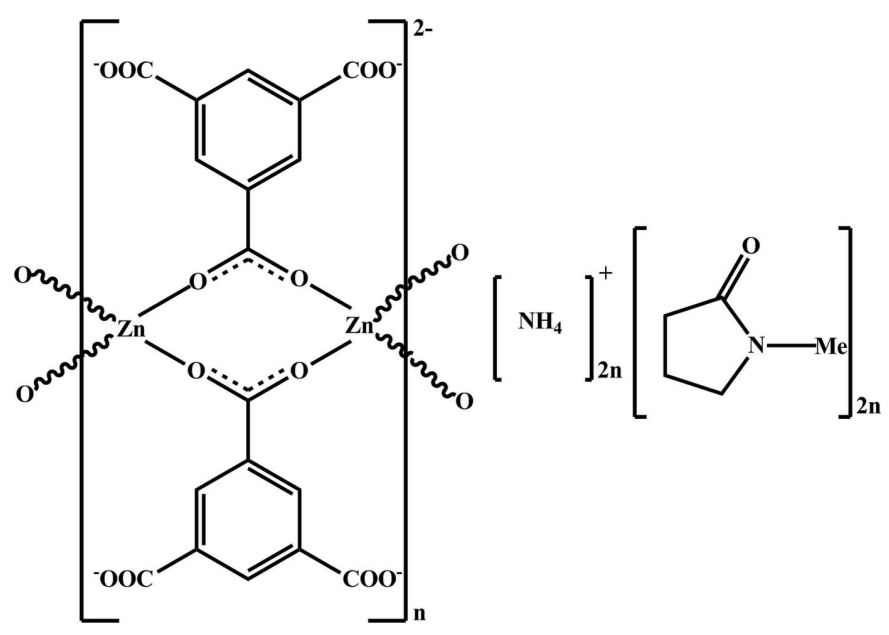

\section{Structural commentary}

The asymmetric unit of the title compound, $\left\{\left(\mathrm{NH}_{4}\right)_{2}\left[\mathrm{Zn}_{2}\left(\mathrm{C}_{9} \mathrm{H}_{3} \mathrm{O}_{6}\right)_{2}\right] \cdot 2 \mathrm{C}_{5} \mathrm{H}_{9} \mathrm{NO}\right\}_{n}$, contains two $\mathrm{Zn}^{\text {II }}$ cations, two ammonium cations, two NMP molecules and two BTC residues (Fig. 1). The compound has a three-dimensional structure constructed from dimeric zinc carboxylate entities and BTC linkers (Fig. 2). The two zinc ions form a unit with six carboxylate units from the two symmetry-independent BTC ligands, and four additional BTC units created by the glide operations and translations. Each of the $\mathrm{Zn}^{\mathrm{II}}$ cations exhibits an $\mathrm{O}_{4}$ coordination set defined by four oxygen atoms of four coordinating BTC residues. The $\mathrm{Zn}-\mathrm{O}$ distances range within 1.927 (5)-1.982 (5) $\AA$ for Zn1 and 1.926 (5)-1.969 (5) $\AA$ for $\mathrm{Zn} 2$. Of the six BTC residues around the $\mathrm{Zn}_{2}$ units, two act in bidentate bridging modes, and combine the two crystallographically unique $\mathrm{Zn}^{\mathrm{II}}$ ions in the binuclear cluster $\left\{\mathrm{Zn}_{2}(\mathrm{COO})_{2}\right\}$ that acts as the SBU in this compound. All of the other carboxylic oxygen atoms coordinate in a monodentate
Table 1

Hydrogen-bond geometry $\left(\AA,^{\circ}\right)$.

\begin{tabular}{lllll}
\hline$D-\mathrm{H} \cdots A$ & $D-\mathrm{H}$ & $\mathrm{H} \cdots A$ & $D \cdots A$ & $D-\mathrm{H} \cdots A$ \\
\hline $\mathrm{N} 3-\mathrm{H} 8 N \cdots \mathrm{O} 1 P$ & $0.89(3)$ & $1.60(7)$ & $2.47(6)$ & $167(7)$ \\
$\mathrm{N} 3-\mathrm{H} 8 N \cdots \mathrm{O} 1 S$ & $0.89(3)$ & $1.91(3)$ & $2.779(9)$ & $166(6)$ \\
$\mathrm{N} 3-\mathrm{H} 7 N \cdots \mathrm{O} 12^{\mathrm{i}}$ & $0.88(3)$ & $1.97(4)$ & $2.786(6)$ & $154(6)$ \\
$\mathrm{N} 3-\mathrm{H} 6 N \cdots \mathrm{O} 9$ & $0.87(3)$ & $2.03(3)$ & $2.867(7)$ & $161(6)$ \\
$\mathrm{N} 3-\mathrm{H} 5 N \cdots \mathrm{O} 4^{\mathrm{ii}}$ & $0.86(3)$ & $1.94(3)$ & $2.800(7)$ & $174(6)$ \\
$\mathrm{N} 2-\mathrm{H} 4 N \cdots \mathrm{O} 13^{\mathrm{iii}}$ & $0.86(3)$ & $1.85(3)$ & $2.713(7)$ & $173(6)$ \\
$\mathrm{N} 2-\mathrm{H} 3 N \cdots \mathrm{O} 11^{\mathrm{i}}$ & $0.88(3)$ & $2.24(4)$ & $3.025(7)$ & $148(6)$ \\
$\mathrm{N} 2-\mathrm{H} 3 N \cdots \mathrm{O} 1^{\mathrm{i}}$ & $0.88(3)$ & $2.41(5)$ & $3.104(7)$ & $136(6)$ \\
$\mathrm{N} 2-\mathrm{H} 2 N \cdots 8^{\mathrm{iv}}$ & $0.88(3)$ & $1.91(4)$ & $2.737(7)$ & $156(6)$ \\
$\mathrm{N} 2-\mathrm{H} 1 N \cdots \mathrm{O} 10^{\mathrm{v}}$ & $0.88(3)$ & $1.97(3)$ & $2.825(7)$ & $163(6)$ \\
\hline
\end{tabular}

Symmetry codes: (i) $x-\frac{1}{2},-y+1, z+\frac{1}{2}$; (ii) $x-1, y, z$; (iii) $x+\frac{1}{2},-y, z+\frac{1}{2}$; (iv) $x+\frac{1}{2},-y+1, z+\frac{1}{2}$; (v) $x, y-1, z$.

fashion (Fig. 1). The $\mathrm{Zn} 1 \cdots \mathrm{Zn} 2$ separation within the $\mathrm{SBU}$ is 3.542 (5) $\AA$. The connection of alternating zinc carboxylate units and BTC linkers results in an infinite three-dimensional $(3,6)$-connected net, which leads to the framework having the same topology as rutile, $\mathrm{TiO}_{2}$.

As a result of the lower symmetry of the SBU, the title compound crystallizes in a reduced symmetry space group $(P n)$ compared to rutile $\left(P 4_{2} / m n m\right)$. Like other $\mathrm{Zn}-\mathrm{BTC}$ frameworks with rtl-topology (Xie et al., 2005; Ordonez et al., 2014), this framework is also porous. There are rectangular channels paralle to the [100] axis, with an approximate dimension of $7.472 \times 9.543 \AA$ in which per asymmetric unit two ammonium cations and two NMP molecules (ordered and disordered ones) reside (Fig. 2). Seven hydrogen-bonding interactions are observed between both of the ammonium cations and the carboxylic framework, $\mathrm{N}$... O distances being in the range $2.713(7)-3.104(7) \AA$; two link each of the
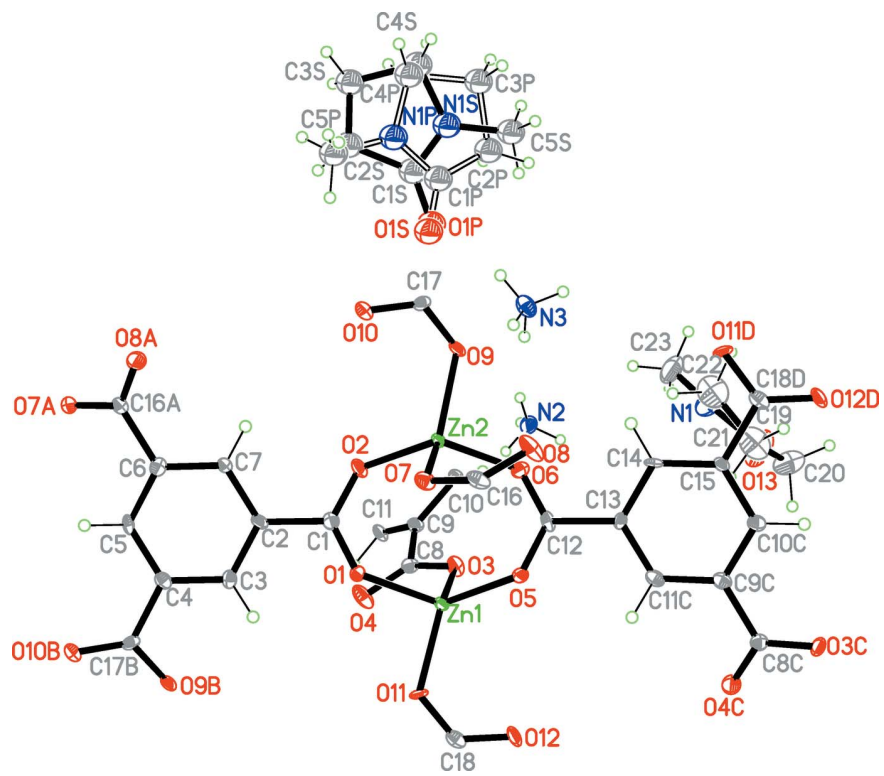

Figure 1

A portion of the crystal structure of the title complex, displaying the atomic labeling. Displacement ellipsoids are drawn at the $50 \%$ probability level. [Symmetry codes: (A) $\frac{1}{2}+x, 2-y, \frac{1}{2}+z$; (B) $1+x, y$, $z$; (C) $x-\frac{1}{2}, 1-y, z-\frac{1}{2}$; (D) $x-1, y, z$.] 


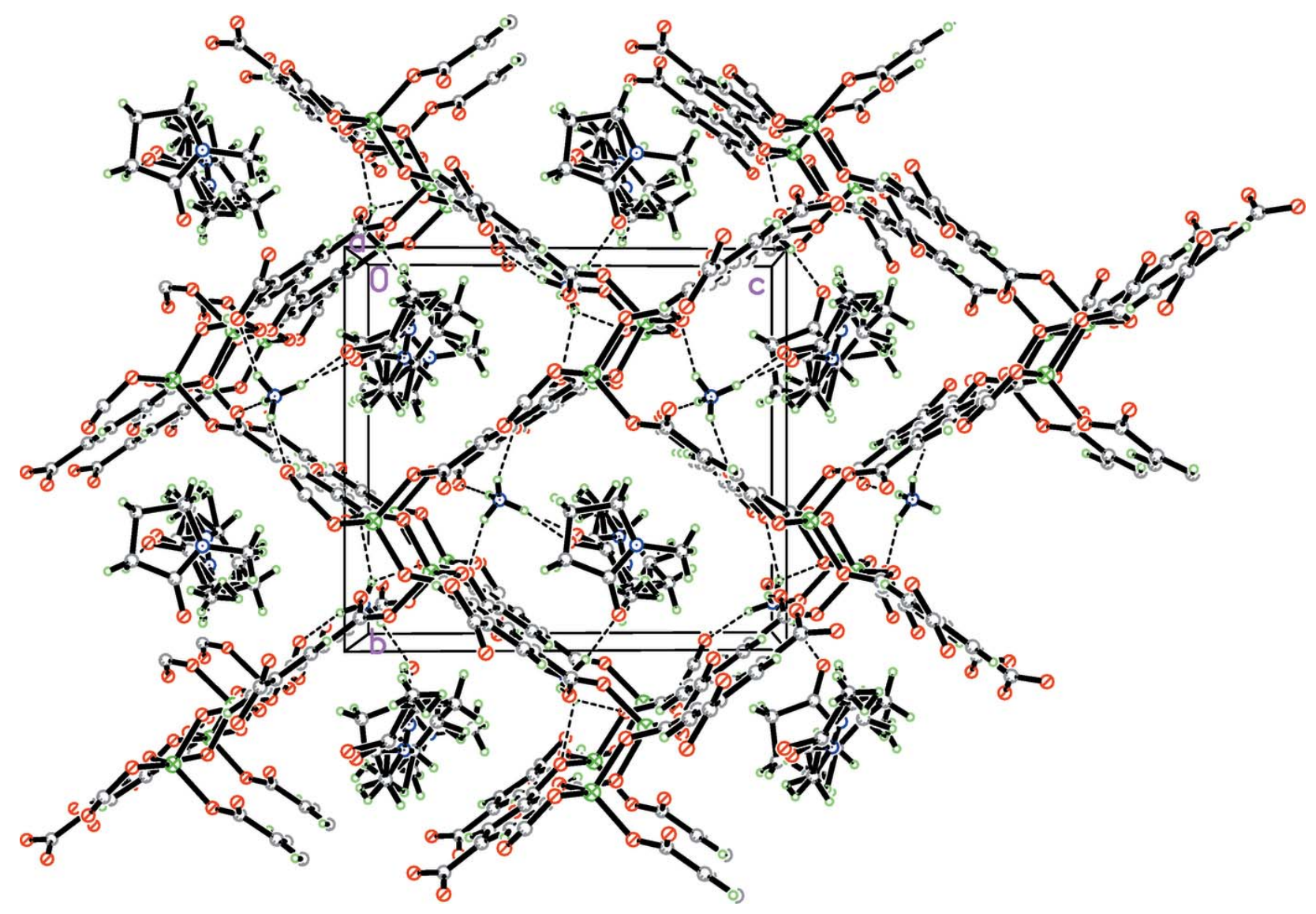

Figure 2

Three-dimensional structure in the unit cell viewed along the $a$ axis. Hydrogen-bonding interactions are shown as dashed lines. C-bound $\mathrm{H}$ atoms in coordination network are omitted for clarity.

ammonium cations with each an NMP molecule (Table 1). The source of the ammonium cations is considered to be from the degradation of di-( $n$-butyl)amine during the reaction.

\section{Database survey}

A literature overview (Xu et al., 2007) reported 41 different Zn-BTC MOFs with a total of 13 types of connectivity modes of BTC with $\mathrm{Zn}$. The 13 modes span all of the possible features of bonds between carboxylic groups and $\mathrm{Zn}$ atoms. Modes with bimetallic $\mathrm{Zn}$ coordination were most frequently found, followed by modes with three $\mathrm{Zn}$ and with four $\mathrm{Zn}$ atoms. A search of the CSD (Groom et al., 2016; ConQuest 1.18, Version 5.37, updates November, 2015) for structures reported after 2007 revealed at least 60 additional $\{\mathrm{Zn}-\mathrm{BTC}\}$ carboxylic networks. The title compound occupies a place in the reticular series of the complexes $\{\mathrm{Zn}-\mathrm{BTC}\}$ Base $\}$ for Base $=\mathrm{Me}_{2} \mathrm{NH}_{2}{ }^{+}$, $\mathrm{Et}_{2} \mathrm{NH}_{2}{ }^{+}, n-\mathrm{Bu}_{2} \mathrm{NH}_{2}{ }^{+}, \mathrm{Et}_{3} \mathrm{NH}^{+},\left(\mathrm{PhCH}_{2}\right) \mathrm{Me}_{3} \mathrm{~N}^{+}$, and $\mathrm{BMIM}=$ 1-butyl-3-methylimidazole (Ordonez et al., 2014). As a result of the size of the templates, the reticular networks differ by the packing modes of the cations in the channels, and correspondingly by channel size within the framework. $\{\mathrm{Zn} / \mathrm{Cd}-$ BTC networks with the same rtl topology have also been reported (Xie et al., 2005; Zhao et al., 2007).

\section{Synthesis and crystallization}

A mixture of $\mathrm{Zn}\left(\mathrm{NO}_{3}\right)_{2} \cdot 6 \mathrm{H}_{2} \mathrm{O}(0.343 \mathrm{~g}, 1.15 \mathrm{mmol}), \mathrm{H}_{3} \mathrm{BTC}$ $(0.244 \mathrm{~g}, 1.16 \mathrm{mmol})$, di-( $n$-butyl)amine $(0.142 \mathrm{~g}, 1.10 \mathrm{mmol})$, and 1-methylpyrrolidin-2-one (NMP, $10 \mathrm{~mL}$ ) was prepared in a capped vial. The solution was transferred to a $23 \mathrm{~mL}$ Teflonlined acid digestion vessel and placed in an oven at $423 \mathrm{~K}$ for four days. The crystals produced were collected in a vial, washed with fresh NMP, and sonicated to remove impurities from the crystals. The main product of the reaction was the MOF $\{\mathrm{Zn}-\mathrm{BTC}\}\left\{n-\mathrm{Bu}_{2} \mathrm{NH}_{2}\right\}$; only few single crystals of the title compound were found as a byproduct. Those crystals were plate shaped and colorless. Synthetic details are given in Ordonez et al. (2014).

\section{Refinement details}

Crystal data, data collection and structure refinement details are summarized in Table 2. C-bound $\mathrm{H}$ atoms were calculated in geometrically idealized positions and refined riding on their parent atoms, with $U_{\text {iso }}(\mathrm{H})=1.2 U_{\text {eq }}(\mathrm{C})$ (aromatic) and $1.5 U_{\text {eq }}(\mathrm{C})$ (methyl), and with $\mathrm{C}-\mathrm{H}=0.95 \AA$ (aromatic) and $0.98 \AA$ (methyl). The methyl $\mathrm{H}$ atoms were allowed to rotate around the corresponding $\mathrm{C}-\mathrm{C}$ bond. $\mathrm{N}$-bound $\mathrm{H}$ atoms in ammonium cations were found in a difference map and refined 
Table 2

Experimental details.

\begin{tabular}{|c|c|}
\hline \multicolumn{2}{|l|}{ Crystal data } \\
\hline Chemical formula & $\left(\mathrm{NH}_{4}\right)_{2}\left[\mathrm{Zn}_{2}\left(\mathrm{C}_{9} \mathrm{H}_{3} \mathrm{O}_{6}\right)_{2}\right] \cdot 2 \mathrm{C}_{5} \mathrm{H}_{9} \mathrm{NO}$ \\
\hline$M_{\mathrm{r}}$ & 779.31 \\
\hline Crystal system, space group & Monoclinic, $P n$ \\
\hline Temperature $(\mathrm{K})$ & 100 \\
\hline$a, b, c(\AA)$ & $9.470(4), 12.351(5), 13.575(5)$ \\
\hline$\beta\left(^{\circ}\right)$ & $94.327(5)$ \\
\hline$V\left(\AA^{3}\right)$ & $1583.2(10)$ \\
\hline$Z$ & 2 \\
\hline Radiation type & Мо $K \alpha$ \\
\hline$\mu\left(\mathrm{mm}^{-1}\right)$ & 1.59 \\
\hline Crystal size $(\mathrm{mm})$ & $0.45 \times 0.35 \times 0.25$ \\
\hline \multicolumn{2}{|l|}{ Data collection } \\
\hline Diffractometer & $\begin{array}{l}\text { Bruker } S M A R T \text { APEXII CCD } \\
\text { area-detector }\end{array}$ \\
\hline Absorption correction & $\begin{array}{l}\text { Multi-scan (SADABS; Bruker, } \\
\text { 2009) }\end{array}$ \\
\hline$T_{\min }, T_{\max }$ & $0.628,0.784$ \\
\hline $\begin{array}{l}\text { No. of measured, independent and } \\
\text { observed }[I>2 \sigma(I)] \text { reflections }\end{array}$ & $13257,6013,5263$ \\
\hline$R_{\text {int }}$ & 0.038 \\
\hline$(\sin \theta / \lambda)_{\max }\left(\AA^{-1}\right)$ & 0.617 \\
\hline \multicolumn{2}{|l|}{ Refinement } \\
\hline$R\left[F^{2}>2 \sigma\left(F^{2}\right)\right], w R\left(F^{2}\right), S$ & $0.033,0.068,0.99$ \\
\hline No. of reflections & 6013 \\
\hline No. of parameters & 525 \\
\hline No. of restraints & 236 \\
\hline H-atom treatment & $\begin{array}{l}\mathrm{H} \text { atoms treated by a mixture of } \\
\text { independent and constrained } \\
\text { refinement }\end{array}$ \\
\hline$\Delta \rho_{\max }, \Delta \rho_{\min }\left(\mathrm{e} \AA^{-3}\right)$ & $0.38,-0.33$ \\
\hline Absolute structure & Refined as an inversion twin. \\
\hline Absolute structure parameter & $0.102(18)$ \\
\hline
\end{tabular}

Computer programs: APEX2 (Bruker, 2014), SAINT-Plus (Bruker, 2009), SHELXTL (Sheldrick, 2008), SHELXL2014 (Sheldrick, 2015), ORTEP-3 for Windows (Farrugia, 2012) and publCIF (Westrip, 2010).

using geometrical restraints to fix the $\mathrm{N}-\mathrm{H}$ distances, and with an isotropic displacement parameter of $U_{\text {iso }}(\mathrm{H})=$ $1.5 U_{\text {eq }}(\mathrm{N})$. One of the NMP molecules is disordered over two positions with partial occupancies 0.903 (8) and 0.097 (8). The geometries of the major and minor NMP moieties were restrained to be similar using a SAME command. The displacement parameters for the disordered NMP molecule were restrained to be similar to each other using a SIMU command with a standard deviation of $0.01 \AA^{2}$.

\section{Acknowledgements}

The authors are grateful for NSF support via DMR-0934212 and DMR-1523611 (PREM), and EPSCoR IIA-1301346.

\section{References}

Almeida Paz, F. A. \& Klinowski, J. (2004). Inorg. Chem. 43, 39483954.

Bruker (2009). SAINT-Plus and SADABS. Bruker AXS Inc., Madison, Wisconsin, USA.

Bruker (2014). APEX2. Bruker AXS Inc., Madison, Wisconsin, USA.

Burrows, A. D., Cassar, K., Friend, R. M. W., Mahon, M. F., Rigby, S. P. \& Warren, J. E. (2005). CrystEngComm, 7, 548-550.

Eddaoudi, M., Moler, D. B., Li, H., Chen, B., Reineke, T. M., O'Keeffe, M. \& Yaghi, O. M. (2001). Acc. Chem. Res. 34, 319-330.

Farrugia, L. J. (2012). J. Appl. Cryst. 45, 849-854.

Gao, J., Ye, K., Yang, L., Xiong, W.-W., Ye, L., Wang, Y. \& Zhang, Q. (2014). Inorg. Chem. 53, 691-693.

Groom, C. R., Bruno, I. J., Lightfoot, M. P. \& Ward, S. C. (2016). Acta Cryst. B72, 171-179.

Hao, X.-R., Wang, X.-L., Shao, K.-Z., Yang, G.-S., Su, Z.-M. \& Yuan, G. (2012). CrystEngComm, 14, 5596-5603.

Liu, Y.-Y., Ma, J.-F., Yang, J. \& Su, Z.-M. (2007). Inorg. Chem. 46, 3027-3037.

Ordonez, C., Fonari, M., Lindline, J., Wei, Q. \& Timofeeva, T. (2014). Cryst. Growth Des. 14, 5452-5465.

Sheldrick, G. M. (2008). Acta Cryst. A64, 112-122.

Sheldrick, G. M. (2015). Acta Cryst. C71, 3-8.

Westrip, S. P. (2010). J. Appl. Cryst. 43, 920-925.

Xie, Y.-M. (2013). J. Solid State Chem. 202, 116-120.

Xie, L., Liu, S., Gao, B., Zhang, C., Sun, C., Li, D. \& Su, Z. (2005). Chem. Commun. pp. 2402-2404.

Xu, L., Choi, E.-Y. \& Kwon, Y.-U. (2007). Inorg. Chem. 46, 1067010680.

Yaghi, O. M., Davis, C. E., Li, G. \& Li, H. (1997). J. Am. Chem. Soc. 119, 2861-2868.

Zhao, J., Zhu, G.-S., Zou, Y.-C., Fang, Q.-R., Xue, M., Li, Z.-Y. \& Qiu, S.-L. (2007). J. Mol. Struct. 871, 80-84. 


\section{supporting information}

Acta Cryst. (2016). E72, 764-767 [doi:10.1107/S2056989016007027]

Crystal structure of poly[bis(ammonium) [bis( $\mu_{4}$-benzene-1,3,5-tricarboxylato)dizincate] 1-methylpyrrolidin-2-one disolvate]

\section{Carlos Ordonez, Marina S. Fonari, Qiang Wei and Tatiana V. Timofeeva}

Computing details

Data collection: APEX2 (Bruker, 2014); cell refinement: SAINT-Plus (Bruker, 2009); data reduction: SAINT-Plus (Bruker, 2009); program(s) used to solve structure: SHELXTL (Sheldrick, 2008); program(s) used to refine structure:

SHELXL2014 (Sheldrick, 2015); molecular graphics: ORTEP-3 for Windows (Farrugia, 2012); software used to prepare material for publication: publCIF (Westrip, 2010).

Poly[bis(ammonium) [bis( $\mu_{4}$-benzene-1,3,5-tricarboxylato)dizincate] 1-methylpyrrolidin-2-one disolvate]

\section{Crystal data}

$\left(\mathrm{NH}_{4}\right)_{2}\left[\mathrm{Zn}_{2}\left(\mathrm{C}_{9} \mathrm{H}_{3} \mathrm{O}_{6}\right)_{2}\right] \cdot 2 \mathrm{C}_{5} \mathrm{H}_{9} \mathrm{NO}$

$M_{r}=779.31$

Monoclinic, $P n$

$a=9.470(4) \AA$

$b=12.351(5) \AA$

$c=13.575(5) \AA$

$\beta=94.327(5)^{\circ}$

$V=1583.2(10) \AA^{3}$

$Z=2$

\section{Data collection}

Bruker SMART APEXII CCD area-detector diffractometer

phi and $\omega$ scans

Absorption correction: multi-scan

(SADABS; Bruker, 2009)

$T_{\min }=0.628, T_{\max }=0.784$

13257 measured reflections

\section{Refinement}

Refinement on $F^{2}$

Least-squares matrix: full

$R\left[F^{2}>2 \sigma\left(F^{2}\right)\right]=0.033$

$w R\left(F^{2}\right)=0.068$

$S=0.99$

6013 reflections

525 parameters

236 restraints

Hydrogen site location: mixed
$F(000)=800$

$D_{\mathrm{x}}=1.635 \mathrm{Mg} \mathrm{m}^{-3}$

Mo $K \alpha$ radiation, $\lambda=0.71073 \AA$

Cell parameters from 3722 reflections

$\theta=4.3-26.2^{\circ}$

$\mu=1.59 \mathrm{~mm}^{-1}$

$T=100 \mathrm{~K}$

Prism, colorless

$0.45 \times 0.35 \times 0.25 \mathrm{~mm}$

6013 independent reflections

5263 reflections with $I>2 \sigma(I)$

$R_{\text {int }}=0.038$

$\theta_{\max }=26.0^{\circ}, \theta_{\min }=4.3^{\circ}$

$h=-11 \rightarrow 11$

$k=-15 \rightarrow 15$

$l=-16 \rightarrow 16$

$\mathrm{H}$ atoms treated by a mixture of independent and constrained refinement

$w=1 /\left[\sigma^{2}\left(F_{\mathrm{o}}^{2}\right)+(0.029 P)^{2}\right]$ where $P=\left(F_{\mathrm{o}}^{2}+2 F_{\mathrm{c}}^{2}\right) / 3$

$(\Delta / \sigma)_{\max }=0.002$

$\Delta \rho_{\max }=0.38 \mathrm{e} \AA^{-3}$

$\Delta \rho_{\min }=-0.33$ e $\AA^{-3}$

Absolute structure: Refined as an inversion twin.

Absolute structure parameter: 0.102 (18) 


\section{Special details}

Geometry. All esds (except the esd in the dihedral angle between two 1.s. planes) are estimated using the full covariance matrix. The cell esds are taken into account individually in the estimation of esds in distances, angles and torsion angles; correlations between esds in cell parameters are only used when they are defined by crystal symmetry. An approximate (isotropic) treatment of cell esds is used for estimating esds involving l.s. planes.

Refinement. Refined as a 2-component inversion twin.

Fractional atomic coordinates and isotropic or equivalent isotropic displacement parameters $\left(\AA^{2}\right)$

\begin{tabular}{|c|c|c|c|c|c|}
\hline & $x$ & $y$ & $z$ & $U_{\text {iso }} * / U_{\text {eq }}$ & Occ. $(<1)$ \\
\hline $\mathrm{Zn} 1$ & $0.89147(5)$ & $0.67955(5)$ & $0.05911(4)$ & 0.00955 (18) & \\
\hline $\mathrm{Zn} 2$ & $0.62372(5)$ & $0.81579(5)$ & $0.17921(4)$ & 0.00980 (19) & \\
\hline $\mathrm{O} 1$ & $0.9514(6)$ & 0.8118 (3) & $0.1336(4)$ & $0.0115(12)$ & \\
\hline $\mathrm{O} 2$ & $0.8070(6)$ & $0.8139(3)$ & $0.2567(4)$ & $0.0138(12)$ & \\
\hline $\mathrm{O} 3$ & $0.8606(5)$ & $0.5625(4)$ & $0.1496(3)$ & $0.0156(11)$ & \\
\hline $\mathrm{O} 4$ & $1.0719(5)$ & $0.5942(4)$ & $0.2254(4)$ & $0.0213(11)$ & \\
\hline $\mathrm{O} 5$ & $0.7069(6)$ & 0.6839 & $-0.0140(4)$ & $0.0126(12)$ & \\
\hline O6 & $0.5633(6)$ & 0.6808 & $0.1113(4)$ & $0.0129(12)$ & \\
\hline O7 & $0.6608(5)$ & $0.9254(4)$ & 0.0835 & $0.0139(10)$ & \\
\hline O8 & $0.4502(5)$ & $0.8860(4)$ & $0.0057(4)$ & $0.0224(12)$ & \\
\hline O9 & $0.4623(5)$ & 0.8233 & $0.2583(4)$ & $0.0127(12)$ & \\
\hline $\mathrm{O} 10$ & $0.5241(4)$ & $0.9792(3)$ & 0.3323 & $0.0177(10)$ & \\
\hline O11 & $1.0529(6)$ & $0.6840(3)$ & $-0.0191(4)$ & $0.0134(12)$ & \\
\hline $\mathrm{O} 12$ & $0.9855(4)$ & $0.5564(3)$ & $-0.1286(3)$ & $0.0193(10)$ & \\
\hline $\mathrm{O} 13$ & $0.2466(6)$ & $0.0795(4)$ & $0.1181(3)$ & $0.0482(14)$ & \\
\hline $\mathrm{C} 1$ & $0.9235(8)$ & $0.8327(5)$ & $0.2208(6)$ & $0.0115(16)$ & \\
\hline $\mathrm{C} 2$ & $1.0366(7)$ & $0.8843(5)$ & $0.2878(5)$ & $0.0127(15)$ & \\
\hline $\mathrm{C} 3$ & $1.1771(7)$ & $0.8723(5)$ & $0.2683(5)$ & $0.0110(15)$ & \\
\hline H3 & 1.2011 & 0.8307 & 0.2132 & $0.013^{*}$ & \\
\hline $\mathrm{C} 4$ & $1.2836(7)$ & $0.9213(5)$ & $0.3297(5)$ & $0.0115(14)$ & \\
\hline $\mathrm{C} 5$ & $1.2461(7)$ & $0.9836(5)$ & $0.4080(5)$ & $0.0113(14)$ & \\
\hline H5 & 1.3177 & 1.0186 & 0.4492 & $0.014^{*}$ & \\
\hline C6 & $1.1059(7)$ & $0.9959(5)$ & $0.4271(5)$ & $0.0134(15)$ & \\
\hline $\mathrm{C} 7$ & $0.9997(8)$ & $0.9460(5)$ & $0.3666(5)$ & $0.0120(15)$ & \\
\hline $\mathrm{H} 7$ & 0.9031 & 0.9544 & 0.3795 & $0.014^{*}$ & \\
\hline $\mathrm{C} 8$ & $0.9530(7)$ & $0.5524(5)$ & $0.2213(5)$ & $0.0128(15)$ & \\
\hline C9 & $0.9120(7)$ & $0.4837(5)$ & $0.3048(5)$ & $0.0134(15)$ & \\
\hline $\mathrm{C} 10$ & $0.7684(8)$ & $0.4637(5)$ & $0.3179(5)$ & $0.0129(14)$ & \\
\hline H10 & 0.6968 & 0.4899 & 0.2710 & $0.015^{*}$ & \\
\hline C11 & $1.0130(8)$ & $0.4415(6)$ & $0.3723(6)$ & $0.0147(15)$ & \\
\hline H11 & 1.1102 & 0.4529 & 0.3628 & $0.018^{*}$ & \\
\hline $\mathrm{C} 12$ & $0.5904(8)$ & $0.6637(5)$ & $0.0231(6)$ & $0.0113(16)$ & \\
\hline $\mathrm{C} 13$ & $0.4770(7)$ & $0.6170(5)$ & $-0.0465(5)$ & $0.0097(15)$ & \\
\hline $\mathrm{C} 14$ & $0.3341(7)$ & $0.6340(5)$ & $-0.0316(5)$ & $0.0114(15)$ & \\
\hline H14 & 0.3079 & 0.6720 & 0.0251 & $0.014^{*}$ & \\
\hline $\mathrm{C} 15$ & $0.2317(7)$ & $0.5949(5)$ & -0.1000 & $0.0108(14)$ & \\
\hline $\mathrm{C} 16$ & $0.5650(8)$ & $0.9342(6)$ & $0.0106(6)$ & $0.0158(16)$ & \\
\hline $\mathrm{C} 17$ & $0.4354(7)$ & $0.9100(5)$ & $0.3065(5)$ & 0.0109 (14) & \\
\hline
\end{tabular}




\begin{tabular}{|c|c|c|c|c|c|}
\hline $\mathrm{C} 18$ & $1.0761(7)$ & $0.6109(5)$ & $-0.0831(5)$ & $0.0133(14)$ & \\
\hline C19 & $0.2289(7)$ & $0.1747(5)$ & $0.0954(5)$ & $0.0292(14)$ & \\
\hline $\mathrm{C} 20$ & $0.1892(9)$ & $0.2178(6)$ & $-0.0067(5)$ & $0.0386(17)$ & \\
\hline $\mathrm{H} 20 \mathrm{~A}$ & 0.0926 & 0.1944 & -0.0305 & $0.046^{*}$ & \\
\hline H20B & 0.2569 & 0.1927 & -0.0539 & $0.046^{*}$ & \\
\hline $\mathrm{C} 21$ & $0.1961(8)$ & $0.3402(6)$ & $0.0056(6)$ & $0.0429(18)$ & \\
\hline $\mathrm{H} 21 \mathrm{~A}$ & 0.2857 & 0.3690 & -0.0170 & $0.051^{*}$ & \\
\hline H21B & 0.1157 & 0.3754 & -0.0328 & $0.051^{*}$ & \\
\hline $\mathrm{C} 22$ & $0.1882(8)$ & $0.3598(5)$ & $0.1150(6)$ & $0.0431(19)$ & \\
\hline $\mathrm{H} 22 \mathrm{~A}$ & 0.2520 & 0.4195 & 0.1385 & $0.052^{*}$ & \\
\hline H22B & 0.0903 & 0.3774 & 0.1306 & $0.052^{*}$ & \\
\hline $\mathrm{C} 23$ & $0.2518(9)$ & $0.2404(6)$ & $0.2649(5)$ & $0.0419(18)$ & \\
\hline $\mathrm{H} 23 \mathrm{~A}$ & 0.1586 & 0.2397 & 0.2918 & $0.063^{*}$ & \\
\hline $\mathrm{H} 23 \mathrm{~B}$ & 0.3087 & 0.2996 & 0.2952 & $0.063 *$ & \\
\hline $\mathrm{H} 23 \mathrm{C}$ & 0.2997 & 0.1713 & 0.2796 & $0.063 *$ & \\
\hline N1 & $0.2347(6)$ & $0.2561(4)$ & 0.1594 (4) & $0.0314(13)$ & \\
\hline N2 & $0.6628(5)$ & $0.0862(4)$ & $0.4962(4)$ & $0.0220(11)$ & \\
\hline $\mathrm{H} 1 \mathrm{~N}$ & $0.628(7)$ & $0.062(5)$ & $0.438(3)$ & $0.033^{*}$ & \\
\hline $\mathrm{H} 2 \mathrm{~N}$ & 0.749 (4) & $0.105(5)$ & $0.483(5)$ & $0.033^{*}$ & \\
\hline $\mathrm{H} 3 \mathrm{~N}$ & $0.620(6)$ & $0.145(4)$ & $0.515(5)$ & $0.033^{*}$ & \\
\hline $\mathrm{H} 4 \mathrm{~N}$ & $0.687(7)$ & $0.036(4)$ & $0.539(4)$ & $0.033^{*}$ & \\
\hline N3 & $0.3320(5)$ & $0.6331(4)$ & $0.3316(4)$ & $0.0197(11)$ & \\
\hline $\mathrm{H} 5 \mathrm{~N}$ & $0.249(4)$ & $0.624(5)$ & $0.302(4)$ & $0.029 *$ & \\
\hline $\mathrm{H} 6 \mathrm{~N}$ & $0.369(6)$ & $0.683(4)$ & $0.296(4)$ & $0.029 *$ & \\
\hline $\mathrm{H} 7 \mathrm{~N}$ & $0.393(6)$ & $0.580(4)$ & $0.328(4)$ & $0.029 *$ & \\
\hline $\mathrm{H} 8 \mathrm{~N}$ & $0.326(7)$ & $0.660(5)$ & $0.392(3)$ & $0.029 *$ & \\
\hline $\mathrm{C} 1 \mathrm{~S}$ & $0.3028(9)$ & $0.7644(7)$ & $0.5883(6)$ & $0.030(2)$ & $0.903(8)$ \\
\hline $\mathrm{C} 2 \mathrm{~S}$ & $0.3572(11)$ & $0.8553(9)$ & $0.6562(9)$ & $0.032(2)$ & $0.903(8)$ \\
\hline $\mathrm{H} 2 \mathrm{~S} 1$ & 0.3523 & 0.9255 & 0.6209 & $0.038^{*}$ & $0.903(8)$ \\
\hline $\mathrm{H} 2 \mathrm{~S} 2$ & 0.4565 & 0.8417 & 0.6812 & $0.038^{*}$ & $0.903(8)$ \\
\hline $\mathrm{C} 3 \mathrm{~S}$ & $0.2619(8)$ & $0.8556(6)$ & $0.7391(5)$ & $0.0324(17)$ & $0.903(8)$ \\
\hline $\mathrm{H} 3 \mathrm{~S} 1$ & 0.3059 & 0.8161 & 0.7970 & $0.039 *$ & $0.903(8)$ \\
\hline $\mathrm{H} 3 \mathrm{~S} 2$ & 0.2409 & 0.9306 & 0.7592 & $0.039^{*}$ & $0.903(8)$ \\
\hline $\mathrm{C} 4 \mathrm{~S}$ & $0.1267(13)$ & $0.7981(13)$ & $0.6975(11)$ & $0.036(2)$ & $0.903(8)$ \\
\hline $\mathrm{H} 4 \mathrm{~S} 1$ & 0.0865 & 0.7520 & 0.7481 & $0.043^{*}$ & $0.903(8)$ \\
\hline $\mathrm{H} 4 \mathrm{~S} 2$ & 0.0541 & 0.8506 & 0.6717 & $0.043^{*}$ & $0.903(8)$ \\
\hline $\mathrm{C} 5 \mathrm{~S}$ & $0.0917(12)$ & $0.6515(11)$ & $0.5659(10)$ & 0.059 (3) & $0.903(8)$ \\
\hline $\mathrm{H} 5 \mathrm{~S} 1$ & 0.0244 & 0.6870 & 0.5178 & $0.089 *$ & $0.903(8)$ \\
\hline H5S2 & 0.0396 & 0.6105 & 0.6132 & $0.089 *$ & $0.903(8)$ \\
\hline $\mathrm{H} 5 \mathrm{~S} 3$ & 0.1522 & 0.6022 & 0.5313 & $0.089^{*}$ & $0.903(8)$ \\
\hline N1S & $0.1793(8)$ & $0.7334(6)$ & $0.6184(5)$ & $0.0355(18)$ & $0.903(8)$ \\
\hline O1S & $0.3602(7)$ & $0.7270(8)$ & $0.5180(5)$ & $0.034(2)$ & $0.903(8)$ \\
\hline $\mathrm{C} 1 \mathrm{P}$ & $0.258(6)$ & $0.737(6)$ & $0.557(4)$ & $0.034(4)$ & $0.097(8)$ \\
\hline $\mathrm{C} 2 \mathrm{P}$ & $0.130(9)$ & $0.665(7)$ & $0.572(8)$ & $0.037(5)$ & $0.097(8)$ \\
\hline $\mathrm{H} 2 \mathrm{P} 1$ & 0.0741 & 0.6512 & 0.5091 & $0.045^{*}$ & $0.097(8)$ \\
\hline $\mathrm{H} 2 \mathrm{P} 2$ & 0.1604 & 0.5951 & 0.6025 & $0.045^{*}$ & $0.097(8)$ \\
\hline C3P & $0.046(5)$ & $0.730(5)$ & $0.641(5)$ & $0.038(5)$ & $0.097(8)$ \\
\hline H3P1 & -0.0295 & 0.7714 & 0.6038 & $0.046^{*}$ & $0.097(8)$ \\
\hline
\end{tabular}




\begin{tabular}{llllll} 
H3P2 & 0.0018 & 0.6813 & 0.6884 & $0.046^{*}$ & $0.097(8)$ \\
C4P & $0.153(10)$ & $0.806(13)$ & $0.696(9)$ & $0.035(4)$ & $0.097(8)$ \\
H4P1 & 0.1800 & 0.7795 & 0.7637 & $0.042^{*}$ & $0.097(8)$ \\
H4P2 & 0.1148 & 0.8802 & 0.6996 & $0.042^{*}$ & $0.097(8)$ \\
C5P & $0.392(9)$ & $0.875(8)$ & $0.650(8)$ & $0.029(9)$ & $0.097(8)$ \\
H5P1 & 0.4522 & 0.8697 & 0.5945 & $0.043^{*}$ & $0.097(8)$ \\
H5P2 & 0.4478 & 0.8561 & 0.7115 & $0.043^{*}$ & $0.097(8)$ \\
H5P3 & 0.3577 & 0.9499 & 0.6548 & $0.043^{*}$ & $0.097(8)$ \\
N1P & $0.272(5)$ & $0.802(4)$ & $0.635(4)$ & $0.032(3)$ & $0.097(8)$ \\
O1P & $0.337(7)$ & $0.732(8)$ & $0.490(4)$ & $0.037(10)$ & $0.097(8)$ \\
\hline
\end{tabular}

Atomic displacement parameters $\left(\AA^{2}\right)$

\begin{tabular}{|c|c|c|c|c|c|c|}
\hline & $U^{11}$ & $U^{22}$ & $U^{\beta 3}$ & $U^{12}$ & $U^{13}$ & $U^{23}$ \\
\hline $\mathrm{Zn} 1$ & 0.0067 (4) & $0.0131(4)$ & 0.0089 (4) & $-0.0007(3)$ & $0.0011(3)$ & $0.0001(3)$ \\
\hline $\mathrm{Zn} 2$ & $0.0068(4)$ & $0.0136(4)$ & $0.0091(4)$ & $-0.0013(3)$ & 0.0008 (3) & $-0.0002(3)$ \\
\hline $\mathrm{O} 1$ & 0.011 (3) & $0.016(3)$ & $0.008(3)$ & $-0.0017(17)$ & $0.000(2)$ & $-0.0005(18)$ \\
\hline $\mathrm{O} 2$ & 0.007 (3) & 0.019 (3) & $0.015(3)$ & $-0.0018(18)$ & $-0.002(2)$ & 0.0015 (19) \\
\hline $\mathrm{O} 3$ & $0.014(3)$ & $0.020(2)$ & $0.013(3)$ & $-0.0026(19)$ & $-0.002(2)$ & $0.007(2)$ \\
\hline $\mathrm{O} 4$ & $0.013(3)$ & $0.026(3)$ & $0.024(3)$ & $-0.0063(19)$ & $-0.001(2)$ & $0.013(2)$ \\
\hline O5 & $0.011(3)$ & 0.019 (3) & 0.008 & $0.0001(19)$ & $0.001(2)$ & $-0.0017(18)$ \\
\hline O6 & $0.014(3)$ & $0.014(3)$ & $0.011(3)$ & $0.0023(17)$ & $0.000(2)$ & $-0.0006(18)$ \\
\hline $\mathrm{O} 7$ & $0.011(3)$ & $0.018(2)$ & $0.013(3)$ & $0.0013(19)$ & $0.000(2)$ & 0.0035 (19) \\
\hline $\mathrm{O} 8$ & $0.011(3)$ & $0.029(3)$ & $0.027(3)$ & $-0.005(2)$ & $-0.001(2)$ & $0.013(2)$ \\
\hline O9 & $0.005(3)$ & $0.014(3)$ & $0.019(3)$ & $0.0011(17)$ & $0.003(2)$ & -0.0007 (19) \\
\hline $\mathrm{O} 10$ & $0.010(2)$ & $0.021(2)$ & $0.022(2)$ & $-0.0048(17)$ & $0.0041(17)$ & $-0.0047(18)$ \\
\hline O11 & 0.015 & $0.017(3)$ & 0.009 (3) & $-0.0019(18)$ & $0.007(2)$ & $-0.0046(18)$ \\
\hline $\mathrm{O} 12$ & $0.007(2)$ & $0.022(2)$ & $0.029(3)$ & $-0.0042(18)$ & $0.0008(18)$ & $-0.010(2)$ \\
\hline $\mathrm{O} 13$ & $0.083(4)$ & $0.022(3)$ & $0.036(3)$ & $0.008(2)$ & -0.015 & $0.001(2)$ \\
\hline $\mathrm{C} 1$ & 0.009 (4) & $0.008(3)$ & $0.017(4)$ & $0.003(3)$ & 0.000 (3) & $0.000(3)$ \\
\hline $\mathrm{C} 2$ & 0.007 (4) & $0.014(3)$ & $0.017(4)$ & $0.000(3)$ & $0.003(3)$ & -0.001 \\
\hline $\mathrm{C} 3$ & $0.013(4)$ & 0.009 (3) & $0.011(4)$ & $-0.002(3)$ & $-0.002(3)$ & $0.002(3)$ \\
\hline $\mathrm{C} 4$ & $0.011(3)$ & 0.011 & $0.013(3)$ & $-0.001(2)$ & $0.001(3)$ & $0.003(3)$ \\
\hline $\mathrm{C} 5$ & 0.009 (3) & $0.013(3)$ & 0.011 & $-0.003(2)$ & -0.005 (2) & $0.001(2)$ \\
\hline C6 & $0.014(4)$ & $0.012(3)$ & $0.013(4)$ & $0.000(3)$ & -0.001 & $0.000(3)$ \\
\hline $\mathrm{C} 7$ & 0.009 (4) & $0.012(3)$ & $0.015(4)$ & -0.001 & $0.002(3)$ & -0.002 \\
\hline $\mathrm{C} 8$ & $0.012(4)$ & $0.015(3)$ & $0.011(3)$ & $0.002(3)$ & $0.001(3)$ & $0.001(3)$ \\
\hline C9 & $0.012(4)$ & $0.014(3)$ & $0.014(4)$ & $0.000(3)$ & $0.003(3)$ & -0.001 \\
\hline $\mathrm{C} 10$ & $0.011(3)$ & $0.013(3)$ & $0.016(3)$ & $-0.004(2)$ & $0.002(2)$ & $0.001(3)$ \\
\hline C11 & $0.009(4)$ & $0.018(3)$ & $0.017(4)$ & -0.003 & 0.001 (3) & $0.001(3)$ \\
\hline $\mathrm{C} 12$ & $0.010(4)$ & 0.011 & $0.012(4)$ & $0.000(3)$ & $-0.006(3)$ & 0.004 (3) \\
\hline $\mathrm{C} 13$ & $0.011(4)$ & $0.011(3)$ & $0.007(4)$ & -0.001 & $-0.002(3)$ & -0.001 \\
\hline $\mathrm{C} 14$ & 0.009 (4) & $0.014(3)$ & $0.013(4)$ & $0.003(3)$ & $0.007(3)$ & -0.001 \\
\hline $\mathrm{C} 15$ & $0.007(3)$ & $0.010(3)$ & $0.015(3)$ & $0.001(2)$ & $0.002(3)$ & -0.002 \\
\hline $\mathrm{C} 16$ & $0.012(4)$ & $0.016(3)$ & $0.020(4)$ & $0.003(3)$ & $0.005(3)$ & $0.004(3)$ \\
\hline $\mathrm{C} 17$ & $0.011(3)$ & $0.016(3)$ & $0.006(3)$ & $0.004(3)$ & $0.000(2)$ & -0.001 \\
\hline $\mathrm{C} 18$ & $0.011(3)$ & $0.013(3)$ & $0.016(3)$ & $0.000(2)$ & $0.002(2)$ & $0.006(3)$ \\
\hline C19 & 0.039 (4) & $0.026(3)$ & $0.022(3)$ & 0.009 (3) & $0.002(3)$ & $-0.003(3)$ \\
\hline
\end{tabular}




$\begin{array}{lllllll}\mathrm{C} 20 & 0.050(5) & 0.040(4) & 0.028(4) & 0.013(4) & 0.010(3) & 0.002(3) \\ \mathrm{C} 21 & 0.036(4) & 0.044(5) & 0.047(5) & 0.001(3) & -0.002(3) & 0.020(4) \\ \mathrm{C} 22 & 0.043(5) & 0.017(3) & 0.069(5) & 0.005(3) & 0.001(4) & -0.002(4) \\ \mathrm{C} 23 & 0.053(5) & 0.047(5) & 0.025(4) & -0.009(4) & 0.001(3) & -0.014(3) \\ \mathrm{N} 1 & 0.045(4) & 0.021(3) & 0.029(3) & -0.001(2) & 0.008(3) & -0.003(2) \\ \mathrm{N} 2 & 0.018(3) & 0.019(3) & 0.028(3) & 0.005(2) & -0.001(2) & -0.011(2) \\ \mathrm{N} 3 & 0.014(3) & 0.018(3) & 0.027(3) & 0.001(2) & -0.001(2) & 0.003(2) \\ \mathrm{C} 1 \mathrm{~S} & 0.026(4) & 0.034(4) & 0.029(4) & 0.005(3) & 0.002(3) & 0.007(4) \\ \mathrm{C} 2 \mathrm{~S} & 0.032(5) & 0.032(5) & 0.030(4) & 0.002(4) & -0.001(4) & -0.002(4) \\ \mathrm{C} 3 \mathrm{~S} & 0.034(4) & 0.034(4) & 0.029(4) & 0.004(3) & 0.003(3) & -0.002(3) \\ \mathrm{C} 4 \mathrm{~S} & 0.032(5) & 0.040(4) & 0.034(4) & 0.007(4) & 0.007(4) & 0.002(4) \\ \mathrm{C} 5 \mathrm{~S} & 0.053(7) & 0.063(7) & 0.059(6) & -0.016(6) & -0.012(6) & -0.015(5) \\ \mathrm{N} 1 \mathrm{~S} & 0.028(4) & 0.041(4) & 0.039(4) & -0.001(3) & 0.005(3) & 0.000(3) \\ \text { O1S } & 0.032(4) & 0.041(4) & 0.030(4) & 0.010(3) & -0.005(3) & -0.008(4) \\ \mathrm{C} 1 \mathrm{P} & 0.028(8) & 0.038(8) & 0.035(8) & 0.004(8) & 0.000(8) & 0.004(8) \\ \mathrm{C} 2 \mathrm{P} & 0.031(9) & 0.042(9) & 0.038(9) & -0.002(9) & 0.001(9) & 0.000(9) \\ \mathrm{C} 3 \mathrm{P} & 0.033(8) & 0.042(8) & 0.039(8) & -0.002(8) & 0.005(8) & 0.002(8) \\ \mathrm{C} 4 \mathrm{P} & 0.031(7) & 0.039(7) & 0.034(7) & 0.003(7) & 0.005(7) & 0.001(7) \\ \mathrm{C} 5 \mathrm{P} & 0.031(15) & 0.033(15) & 0.022(15) & 0.005(15) & -0.004(15) & -0.003(14) \\ \mathrm{N} 1 \mathrm{P} & 0.030(6) & 0.035(6) & 0.032(6) & 0.004(6) & 0.001(6) & 0.001(6) \\ \text { O1P } & 0.041(17) & 0.038(16) & 0.032(17) & 0.017(16) & -0.006(16) & -0.001(17) \\ & & & & & & \end{array}$

Geometric parameters $\left(\AA,{ }^{\circ}\right)$

\begin{tabular}{llll}
\hline $\mathrm{Zn} 1-\mathrm{O} 3$ & $1.933(5)$ & $\mathrm{C} 20-\mathrm{H} 20 \mathrm{~A}$ & 0.9900 \\
$\mathrm{Zn} 1-\mathrm{O} 11$ & $1.927(5)$ & $\mathrm{C} 2-\mathrm{H} 20 \mathrm{~B}$ & 0.9900 \\
$\mathrm{Zn} 1-\mathrm{O} 5$ & $1.944(5)$ & $\mathrm{C} 21-\mathrm{C} 22$ & $1.513(11)$ \\
$\mathrm{Zn} 1-\mathrm{O} 1$ & $1.982(5)$ & $\mathrm{C} 21-\mathrm{H} 21 \mathrm{~A}$ & 0.9900 \\
$\mathrm{Zn} 2-\mathrm{O} 7$ & $1.926(5)$ & $\mathrm{C} 21-\mathrm{H} 21 \mathrm{~B}$ & 0.9900 \\
$\mathrm{Zn} 2-\mathrm{O} 9$ & $1.935(5)$ & $\mathrm{C} 22-\mathrm{N} 1$ & $1.469(8)$ \\
$\mathrm{Zn} 2-\mathrm{O} 2$ & $1.960(5)$ & $\mathrm{C} 22-\mathrm{H} 22 \mathrm{~A}$ & 0.9900 \\
$\mathrm{Zn} 2-\mathrm{O} 6$ & $1.969(5)$ & $\mathrm{C} 22-\mathrm{H} 22 \mathrm{~B}$ & 0.9900 \\
$\mathrm{O} 1-\mathrm{C} 1$ & $1.259(9)$ & $\mathrm{C} 23-\mathrm{N} 1$ & $1.443(9)$ \\
$\mathrm{O} 2-\mathrm{C} 1$ & $1.261(9)$ & $\mathrm{C} 23-\mathrm{H} 23 \mathrm{~A}$ & 0.9800 \\
$\mathrm{O} 3-\mathrm{C} 8$ & $1.265(8)$ & $\mathrm{C} 23-\mathrm{H} 23 \mathrm{~B}$ & 0.9800 \\
$\mathrm{O} 4-\mathrm{C} 8$ & $1.237(8)$ & $\mathrm{C} 23-\mathrm{H} 23 \mathrm{C}$ & 0.9800 \\
$\mathrm{O} 5-\mathrm{C} 12$ & $1.272(9)$ & $\mathrm{N} 2-\mathrm{H} 1 \mathrm{~N}$ & $0.88(3)$ \\
$\mathrm{O} 6-\mathrm{C} 12$ & $1.261(9)$ & $\mathrm{N} 2-\mathrm{H} 2 \mathrm{~N}$ & $0.88(3)$ \\
$\mathrm{O} 7-\mathrm{C} 16$ & $1.296(8)$ & $\mathrm{N} 2-\mathrm{H} 3 \mathrm{~N}$ & $0.88(3)$ \\
$\mathrm{O} 8-\mathrm{C} 16$ & $1.237(8)$ & $\mathrm{N} 2-\mathrm{H} 4 \mathrm{~N}$ & $0.86(3)$ \\
$\mathrm{O} 9-\mathrm{C} 17$ & $1.290(7)$ & $\mathrm{N} 3-\mathrm{H} 5 \mathrm{~N}$ & $0.86(3)$ \\
$\mathrm{O} 10-\mathrm{C} 17$ & $1.231(7)$ & $\mathrm{N} 3-\mathrm{H} 6 \mathrm{~N}$ & $0.87(3)$ \\
$\mathrm{O} 11-\mathrm{C} 18$ & $1.283(8)$ & $\mathrm{N} 3-\mathrm{H} 7 \mathrm{~N}$ & $0.88(3)$ \\
$\mathrm{O} 12-\mathrm{C} 18$ & $1.222(8)$ & $\mathrm{N} 3-\mathrm{H} 8 \mathrm{~N}$ & $0.89(3)$ \\
$\mathrm{O} 13-\mathrm{C} 19$ & $1.224(7)$ & $\mathrm{C} 1 \mathrm{~S}-\mathrm{O} 1 \mathrm{~S}$ & $1.223(11)$ \\
$\mathrm{C} 1-\mathrm{C} 2$ & $1.494(10)$ & $\mathrm{C} 1 \mathrm{~S}-\mathrm{N} 1 \mathrm{~S}$ & $1.325(8)$ \\
$\mathrm{C} 2-\mathrm{C} 7$ & $1.381(9)$ & $\mathrm{C} 2 \mathrm{~S}-\mathrm{C} 3 \mathrm{C}$ & $1.517(14)$ \\
$\mathrm{C} 2-\mathrm{C} 3$ & $1.384(9)$ & & $1.495(13)$ \\
& &
\end{tabular}




\begin{tabular}{|c|c|c|c|}
\hline $\mathrm{C} 3-\mathrm{C} 4$ & $1.397(9)$ & $\mathrm{C} 2 \mathrm{~S}-\mathrm{H} 2 \mathrm{~S} 1$ & 0.9900 \\
\hline $\mathrm{C} 3-\mathrm{H} 3$ & 0.9500 & $\mathrm{C} 2 \mathrm{~S}-\mathrm{H} 2 \mathrm{~S} 2$ & 0.9900 \\
\hline $\mathrm{C} 4-\mathrm{C} 5$ & $1.380(9)$ & $\mathrm{C} 3 \mathrm{~S}-\mathrm{C} 4 \mathrm{~S}$ & $1.533(13)$ \\
\hline $\mathrm{C} 4-\mathrm{C} 17^{\mathrm{i}}$ & $1.501(9)$ & $\mathrm{C} 3 \mathrm{~S}-\mathrm{H} 3 \mathrm{~S} 1$ & 0.9900 \\
\hline $\mathrm{C} 5-\mathrm{C} 6$ & $1.381(9)$ & $\mathrm{C} 3 \mathrm{~S}-\mathrm{H} 3 \mathrm{~S} 2$ & 0.9900 \\
\hline $\mathrm{C} 5-\mathrm{H} 5$ & 0.9500 & $\mathrm{C} 4 \mathrm{~S}-\mathrm{N} 1 \mathrm{~S}$ & $1.457(11)$ \\
\hline $\mathrm{C} 6-\mathrm{C} 7$ & $1.392(10)$ & $\mathrm{C} 4 \mathrm{~S}-\mathrm{H} 4 \mathrm{~S} 1$ & 0.9900 \\
\hline $\mathrm{C} 6-\mathrm{C} 16^{\mathrm{ii}}$ & $1.500(10)$ & $\mathrm{C} 4 \mathrm{~S}-\mathrm{H} 4 \mathrm{~S} 2$ & 0.9900 \\
\hline $\mathrm{C} 7-\mathrm{H} 7$ & 0.9500 & $\mathrm{C} 5 \mathrm{~S}-\mathrm{N} 1 \mathrm{~S}$ & $1.459(11)$ \\
\hline $\mathrm{C} 8-\mathrm{C} 9$ & $1.490(9)$ & $\mathrm{C} 5 \mathrm{~S}-\mathrm{H} 5 \mathrm{~S} 1$ & 0.9800 \\
\hline $\mathrm{C} 9-\mathrm{C} 11$ & $1.376(10)$ & $\mathrm{C} 5 \mathrm{~S}-\mathrm{H} 5 \mathrm{~S} 2$ & 0.9800 \\
\hline $\mathrm{C} 9-\mathrm{C} 10$ & $1.407(9)$ & $\mathrm{C} 5 \mathrm{~S}-\mathrm{H} 5 \mathrm{~S} 3$ & 0.9800 \\
\hline $\mathrm{C} 10-\mathrm{C} 15^{\mathrm{iii}}$ & $1.395(9)$ & $\mathrm{C} 1 \mathrm{P}-\mathrm{O} 1 \mathrm{P}$ & $1.22(3)$ \\
\hline $\mathrm{C} 10-\mathrm{H} 10$ & 0.9500 & $\mathrm{C} 1 \mathrm{P}-\mathrm{N} 1 \mathrm{P}$ & $1.33(3)$ \\
\hline $\mathrm{C} 11-\mathrm{C} 13^{\mathrm{iii}}$ & $1.382(9)$ & $\mathrm{C} 1 \mathrm{P}-\mathrm{C} 2 \mathrm{P}$ & $1.52(3)$ \\
\hline $\mathrm{C} 11-\mathrm{H} 11$ & 0.9500 & $\mathrm{C} 2 \mathrm{P}-\mathrm{C} 3 \mathrm{P}$ & $1.50(3)$ \\
\hline $\mathrm{C} 12-\mathrm{C} 13$ & $1.492(10)$ & $\mathrm{C} 2 \mathrm{P}-\mathrm{H} 2 \mathrm{P} 1$ & 0.9900 \\
\hline $\mathrm{C} 13-\mathrm{C} 11^{\mathrm{iv}}$ & $1.382(9)$ & $\mathrm{C} 2 \mathrm{P}-\mathrm{H} 2 \mathrm{P} 2$ & 0.9900 \\
\hline $\mathrm{C} 13-\mathrm{C} 14$ & $1.400(9)$ & $\mathrm{C} 3 \mathrm{P}-\mathrm{C} 4 \mathrm{P}$ & $1.53(3)$ \\
\hline $\mathrm{C} 14-\mathrm{C} 15$ & $1.378(9)$ & $\mathrm{C} 3 \mathrm{P}-\mathrm{H} 3 \mathrm{P} 1$ & 0.9900 \\
\hline $\mathrm{C} 14-\mathrm{H} 14$ & 0.9500 & $\mathrm{C} 3 \mathrm{P}-\mathrm{H} 3 \mathrm{P} 2$ & 0.9900 \\
\hline $\mathrm{C} 15-\mathrm{C} 10^{\mathrm{iv}}$ & $1.395(9)$ & $\mathrm{C} 4 \mathrm{P}-\mathrm{N} 1 \mathrm{P}$ & $1.46(3)$ \\
\hline $\mathrm{C} 15-\mathrm{C} 18^{\mathrm{v}}$ & $1.521(9)$ & $\mathrm{C} 4 \mathrm{P}-\mathrm{H} 4 \mathrm{P} 1$ & 0.9900 \\
\hline $\mathrm{C} 16-\mathrm{C}^{\mathrm{vi}}$ & $1.500(10)$ & $\mathrm{C} 4 \mathrm{P}-\mathrm{H} 4 \mathrm{P} 2$ & 0.9900 \\
\hline $\mathrm{C} 17-\mathrm{C} 4^{\mathrm{v}}$ & $1.501(9)$ & $\mathrm{C} 5 \mathrm{P}-\mathrm{N} 1 \mathrm{P}$ & $1.46(3)$ \\
\hline $\mathrm{C} 18-\mathrm{C} 15^{\mathrm{i}}$ & $1.521(9)$ & $\mathrm{C} 5 \mathrm{P}-\mathrm{H} 5 \mathrm{P} 1$ & 0.9800 \\
\hline $\mathrm{C} 19-\mathrm{N} 1$ & $1.327(7)$ & $\mathrm{C} 5 \mathrm{P}-\mathrm{H} 5 \mathrm{P} 2$ & 0.9800 \\
\hline $\mathrm{C} 19-\mathrm{C} 20$ & $1.505(9)$ & $\mathrm{C} 5 \mathrm{P}-\mathrm{H} 5 \mathrm{P} 3$ & 0.9800 \\
\hline $\mathrm{C} 20-\mathrm{C} 21$ & $1.522(9)$ & & \\
\hline $\mathrm{O} 3-\mathrm{Zn} 1-\mathrm{O} 11$ & $122.4(2)$ & $\mathrm{H} 21 \mathrm{~A}-\mathrm{C} 21-\mathrm{H} 21 \mathrm{~B}$ & 108.8 \\
\hline $\mathrm{O} 3-\mathrm{Zn} 1-\mathrm{O} 5$ & $99.9(2)$ & $\mathrm{N} 1-\mathrm{C} 22-\mathrm{C} 21$ & $103.1(5)$ \\
\hline $\mathrm{O} 11-\mathrm{Zn} 1-\mathrm{O} 5$ & $116.0(2)$ & $\mathrm{N} 1-\mathrm{C} 22-\mathrm{H} 22 \mathrm{~A}$ & 111.1 \\
\hline $\mathrm{O} 3-\mathrm{Zn} 1-\mathrm{O} 1$ & $110.1(2)$ & $\mathrm{C} 21-\mathrm{C} 22-\mathrm{H} 22 \mathrm{~A}$ & 111.1 \\
\hline $\mathrm{O} 11-\mathrm{Zn} 1-\mathrm{O} 1$ & $92.8(2)$ & $\mathrm{N} 1-\mathrm{C} 22-\mathrm{H} 22 \mathrm{~B}$ & 111.1 \\
\hline $\mathrm{O} 5-\mathrm{Zn} 1-\mathrm{O} 1$ & $116.7(2)$ & $\mathrm{C} 21-\mathrm{C} 22-\mathrm{H} 22 \mathrm{~B}$ & 111.1 \\
\hline $\mathrm{O} 7-\mathrm{Zn} 2-\mathrm{O} 9$ & $122.16(19)$ & $\mathrm{H} 22 \mathrm{~A}-\mathrm{C} 22-\mathrm{H} 22 \mathrm{~B}$ & 109.1 \\
\hline $\mathrm{O} 7-\mathrm{Zn} 2-\mathrm{O} 2$ & $99.8(2)$ & $\mathrm{N} 1-\mathrm{C} 23-\mathrm{H} 23 \mathrm{~A}$ & 109.5 \\
\hline $\mathrm{O} 9-\mathrm{Zn} 2-\mathrm{O} 2$ & $114.1(2)$ & $\mathrm{N} 1-\mathrm{C} 23-\mathrm{H} 23 \mathrm{~B}$ & 109.5 \\
\hline $\mathrm{O} 7-\mathrm{Zn} 2-\mathrm{O} 6$ & $109.9(2)$ & $\mathrm{H} 23 \mathrm{~A}-\mathrm{C} 23-\mathrm{H} 23 \mathrm{~B}$ & 109.5 \\
\hline $\mathrm{O} 9-\mathrm{Zn} 2-\mathrm{O} 6$ & $94.9(2)$ & $\mathrm{N} 1-\mathrm{C} 23-\mathrm{H} 23 \mathrm{C}$ & 109.5 \\
\hline $\mathrm{O} 2-\mathrm{Zn} 2-\mathrm{O} 6$ & $117.1(2)$ & $\mathrm{H} 23 \mathrm{~A}-\mathrm{C} 23-\mathrm{H} 23 \mathrm{C}$ & 109.5 \\
\hline $\mathrm{C} 1-\mathrm{O} 1-\mathrm{Zn} 1$ & $125.2(5)$ & $\mathrm{H} 23 \mathrm{~B}-\mathrm{C} 23-\mathrm{H} 23 \mathrm{C}$ & 109.5 \\
\hline $\mathrm{C} 1-\mathrm{O} 2-\mathrm{Zn} 2$ & $123.8(5)$ & $\mathrm{C} 19-\mathrm{N} 1-\mathrm{C} 23$ & $123.0(6)$ \\
\hline $\mathrm{C} 8-\mathrm{O} 3-\mathrm{Zn} 1$ & $115.7(4)$ & $\mathrm{C} 19-\mathrm{N} 1-\mathrm{C} 22$ & $113.3(5)$ \\
\hline $\mathrm{C} 12-\mathrm{O} 5-\mathrm{Zn} 1$ & $124.5(5)$ & $\mathrm{C} 23-\mathrm{N} 1-\mathrm{C} 22$ & $122.1(6)$ \\
\hline $\mathrm{C} 12-\mathrm{O} 6-\mathrm{Zn} 2$ & $120.9(5)$ & $\mathrm{H} 1 \mathrm{~N}-\mathrm{N} 2-\mathrm{H} 2 \mathrm{~N}$ & $101(6)$ \\
\hline $\mathrm{C} 16-\mathrm{O} 7-\mathrm{Zn} 2$ & $114.9(4)$ & $\mathrm{H} 1 \mathrm{~N}-\mathrm{N} 2-\mathrm{H} 3 \mathrm{~N}$ & $113(6)$ \\
\hline
\end{tabular}




\begin{tabular}{|c|c|c|c|}
\hline $\mathrm{C} 17-\mathrm{O} 9-\mathrm{Zn} 2$ & $121.0(4)$ & $\mathrm{H} 2 \mathrm{~N}-\mathrm{N} 2-\mathrm{H} 3 \mathrm{~N}$ & $107(6)$ \\
\hline $\mathrm{C} 18-\mathrm{O} 11-\mathrm{Zn} 1$ & $122.4(4)$ & $\mathrm{H} 1 \mathrm{~N}-\mathrm{N} 2-\mathrm{H} 4 \mathrm{~N}$ & $115(6)$ \\
\hline $\mathrm{O} 1-\mathrm{C} 1-\mathrm{O} 2$ & $125.3(7)$ & $\mathrm{H} 2 \mathrm{~N}-\mathrm{N} 2-\mathrm{H} 4 \mathrm{~N}$ & $97(6)$ \\
\hline $\mathrm{O} 1-\mathrm{C} 1-\mathrm{C} 2$ & $117.9(7)$ & $\mathrm{H} 3 \mathrm{~N}-\mathrm{N} 2-\mathrm{H} 4 \mathrm{~N}$ & $120(6)$ \\
\hline $\mathrm{O} 2-\mathrm{C} 1-\mathrm{C} 2$ & $116.8(7)$ & $\mathrm{H} 5 \mathrm{~N}-\mathrm{N} 3-\mathrm{H} 6 \mathrm{~N}$ & $103(6)$ \\
\hline $\mathrm{C} 7-\mathrm{C} 2-\mathrm{C} 3$ & $120.7(6)$ & $\mathrm{H} 5 \mathrm{~N}-\mathrm{N} 3-\mathrm{H} 7 \mathrm{~N}$ & $117(6)$ \\
\hline $\mathrm{C} 7-\mathrm{C} 2-\mathrm{C} 1$ & $119.7(7)$ & $\mathrm{H} 6 \mathrm{~N}-\mathrm{N} 3-\mathrm{H} 7 \mathrm{~N}$ & $102(6)$ \\
\hline $\mathrm{C} 3-\mathrm{C} 2-\mathrm{C} 1$ & $119.6(6)$ & $\mathrm{H} 5 \mathrm{~N}-\mathrm{N} 3-\mathrm{H} 8 \mathrm{~N}$ & $111(6)$ \\
\hline $\mathrm{C} 2-\mathrm{C} 3-\mathrm{C} 4$ & $120.1(6)$ & $\mathrm{H} 6 \mathrm{~N}-\mathrm{N} 3-\mathrm{H} 8 \mathrm{~N}$ & $107(6)$ \\
\hline $\mathrm{C} 2-\mathrm{C} 3-\mathrm{H} 3$ & 120.0 & $\mathrm{H} 7 \mathrm{~N}-\mathrm{N} 3-\mathrm{H} 8 \mathrm{~N}$ & $115(6)$ \\
\hline $\mathrm{C} 4-\mathrm{C} 3-\mathrm{H} 3$ & 120.0 & $\mathrm{O} 1 \mathrm{~S}-\mathrm{C} 1 \mathrm{~S}-\mathrm{N} 1 \mathrm{~S}$ & $125.9(9)$ \\
\hline $\mathrm{C} 5-\mathrm{C} 4-\mathrm{C} 3$ & $119.0(7)$ & $\mathrm{O} 1 \mathrm{~S}-\mathrm{C} 1 \mathrm{~S}-\mathrm{C} 2 \mathrm{~S}$ & $127.0(9)$ \\
\hline $\mathrm{C} 5-\mathrm{C} 4-\mathrm{C} 17^{\mathrm{i}}$ & $121.3(6)$ & $\mathrm{N} 1 \mathrm{~S}-\mathrm{C} 1 \mathrm{~S}-\mathrm{C} 2 \mathrm{~S}$ & $107.1(7)$ \\
\hline $\mathrm{C} 3-\mathrm{C} 4-\mathrm{C} 17^{\mathrm{i}}$ & $119.6(6)$ & $\mathrm{C} 1 \mathrm{~S}-\mathrm{C} 2 \mathrm{~S}-\mathrm{C} 3 \mathrm{~S}$ & $105.2(7)$ \\
\hline $\mathrm{C} 4-\mathrm{C} 5-\mathrm{C} 6$ & $120.9(6)$ & $\mathrm{C} 1 \mathrm{~S}-\mathrm{C} 2 \mathrm{~S}-\mathrm{H} 2 \mathrm{~S} 1$ & 110.7 \\
\hline $\mathrm{C} 4-\mathrm{C} 5-\mathrm{H} 5$ & 119.6 & $\mathrm{C} 3 \mathrm{~S}-\mathrm{C} 2 \mathrm{~S}-\mathrm{H} 2 \mathrm{~S} 1$ & 110.7 \\
\hline $\mathrm{C} 6-\mathrm{C} 5-\mathrm{H} 5$ & 119.6 & $\mathrm{C} 1 \mathrm{~S}-\mathrm{C} 2 \mathrm{~S}-\mathrm{H} 2 \mathrm{~S} 2$ & 110.7 \\
\hline $\mathrm{C} 7-\mathrm{C} 6-\mathrm{C} 5$ & $120.2(6)$ & $\mathrm{C} 3 \mathrm{~S}-\mathrm{C} 2 \mathrm{~S}-\mathrm{H} 2 \mathrm{~S} 2$ & 110.7 \\
\hline $\mathrm{C} 7-\mathrm{C} 6-\mathrm{C} 16^{\mathrm{ii}}$ & $119.0(6)$ & $\mathrm{H} 2 \mathrm{~S} 1-\mathrm{C} 2 \mathrm{~S}-\mathrm{H} 2 \mathrm{~S} 2$ & 108.8 \\
\hline $\mathrm{C} 5-\mathrm{C} 6-\mathrm{C} 16^{\mathrm{ii}}$ & $120.8(6)$ & $\mathrm{C} 4 \mathrm{~S}-\mathrm{C} 3 \mathrm{~S}-\mathrm{C} 2 \mathrm{~S}$ & $104.9(7)$ \\
\hline $\mathrm{C} 2-\mathrm{C} 7-\mathrm{C} 6$ & $119.2(7)$ & $\mathrm{C} 4 \mathrm{~S}-\mathrm{C} 3 \mathrm{~S}-\mathrm{H} 3 \mathrm{~S} 1$ & 110.8 \\
\hline $\mathrm{C} 2-\mathrm{C} 7-\mathrm{H} 7$ & 120.4 & $\mathrm{C} 2 \mathrm{~S}-\mathrm{C} 3 \mathrm{~S}-\mathrm{H} 3 \mathrm{~S} 1$ & 110.8 \\
\hline $\mathrm{C} 6-\mathrm{C} 7-\mathrm{H} 7$ & 120.4 & $\mathrm{C} 4 \mathrm{~S}-\mathrm{C} 3 \mathrm{~S}-\mathrm{H} 3 \mathrm{~S} 2$ & 110.8 \\
\hline $\mathrm{O} 4-\mathrm{C} 8-\mathrm{O} 3$ & $124.6(6)$ & $\mathrm{C} 2 \mathrm{~S}-\mathrm{C} 3 \mathrm{~S}-\mathrm{H} 3 \mathrm{~S} 2$ & 110.8 \\
\hline $\mathrm{O} 4-\mathrm{C} 8-\mathrm{C} 9$ & $119.5(6)$ & $\mathrm{H} 3 \mathrm{~S} 1-\mathrm{C} 3 \mathrm{~S}-\mathrm{H} 3 \mathrm{~S} 2$ & 108.8 \\
\hline $\mathrm{O} 3-\mathrm{C} 8-\mathrm{C} 9$ & $115.9(6)$ & $\mathrm{N} 1 \mathrm{~S}-\mathrm{C} 4 \mathrm{~S}-\mathrm{C} 3 \mathrm{~S}$ & $101.7(7)$ \\
\hline $\mathrm{C} 11-\mathrm{C} 9-\mathrm{C} 10$ & $118.6(7)$ & $\mathrm{N} 1 \mathrm{~S}-\mathrm{C} 4 \mathrm{~S}-\mathrm{H} 4 \mathrm{~S} 1$ & 111.4 \\
\hline $\mathrm{C} 11-\mathrm{C} 9-\mathrm{C} 8$ & $120.9(7)$ & $\mathrm{C} 3 \mathrm{~S}-\mathrm{C} 4 \mathrm{~S}-\mathrm{H} 4 \mathrm{~S} 1$ & 111.4 \\
\hline $\mathrm{C} 10-\mathrm{C} 9-\mathrm{C} 8$ & $120.4(6)$ & $\mathrm{N} 1 \mathrm{~S}-\mathrm{C} 4 \mathrm{~S}-\mathrm{H} 4 \mathrm{~S} 2$ & 111.4 \\
\hline $\mathrm{C} 15^{\mathrm{iii}}-\mathrm{C} 10-\mathrm{C} 9$ & $119.6(6)$ & $\mathrm{C} 3 \mathrm{~S}-\mathrm{C} 4 \mathrm{~S}-\mathrm{H} 4 \mathrm{~S} 2$ & 111.4 \\
\hline $\mathrm{C} 15^{\mathrm{iii}}-\mathrm{C} 10-\mathrm{H} 10$ & 120.2 & $\mathrm{H} 4 \mathrm{~S} 1-\mathrm{C} 4 \mathrm{~S}-\mathrm{H} 4 \mathrm{~S} 2$ & 109.3 \\
\hline $\mathrm{C} 9-\mathrm{C} 10-\mathrm{H} 10$ & 120.2 & $\mathrm{~N} 1 \mathrm{~S}-\mathrm{C} 5 \mathrm{~S}-\mathrm{H} 5 \mathrm{~S} 1$ & 109.5 \\
\hline $\mathrm{C} 13^{\mathrm{iii}}-\mathrm{C} 11-\mathrm{C} 9$ & $121.9(7)$ & $\mathrm{N} 1 \mathrm{~S}-\mathrm{C} 5 \mathrm{~S}-\mathrm{H} 5 \mathrm{~S} 2$ & 109.5 \\
\hline 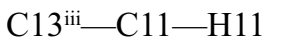 & 119.0 & $\mathrm{H} 5 \mathrm{~S} 1-\mathrm{C} 5 \mathrm{~S}-\mathrm{H} 5 \mathrm{~S} 2$ & 109.5 \\
\hline C9- $111-\mathrm{H} 11$ & 119.0 & $\mathrm{~N} 1 \mathrm{~S}-\mathrm{C} 5 \mathrm{~S}-\mathrm{H} 5 \mathrm{~S} 3$ & 109.5 \\
\hline $\mathrm{O} 5-\mathrm{C} 12-\mathrm{O} 6$ & $126.0(7)$ & $\mathrm{H} 5 \mathrm{~S} 1-\mathrm{C} 5 \mathrm{~S}-\mathrm{H} 5 \mathrm{~S} 3$ & 109.5 \\
\hline $\mathrm{O} 5-\mathrm{C} 12-\mathrm{C} 13$ & $115.4(7)$ & $\mathrm{H} 5 \mathrm{~S} 2-\mathrm{C} 5 \mathrm{~S}-\mathrm{H} 5 \mathrm{~S} 3$ & 109.5 \\
\hline $\mathrm{O} 6-\mathrm{C} 12-\mathrm{C} 13$ & $118.5(7)$ & $\mathrm{C} 1 \mathrm{~S}-\mathrm{N} 1 \mathrm{~S}-\mathrm{C} 5 \mathrm{~S}$ & $122.2(8)$ \\
\hline $\mathrm{C} 11^{\mathrm{iv}}-\mathrm{C} 13-\mathrm{C} 14$ & $119.6(6)$ & $\mathrm{C} 1 \mathrm{~S}-\mathrm{N} 1 \mathrm{~S}-\mathrm{C} 4 \mathrm{~S}$ & $115.4(8)$ \\
\hline $\mathrm{C} 11^{\mathrm{iv}}-\mathrm{C} 13-\mathrm{C} 12$ & $119.9(7)$ & $\mathrm{C} 5 \mathrm{~S}-\mathrm{N} 1 \mathrm{~S}-\mathrm{C} 4 \mathrm{~S}$ & $121.8(8)$ \\
\hline $\mathrm{C} 14-\mathrm{C} 13-\mathrm{C} 12$ & $120.5(6)$ & $\mathrm{O} 1 \mathrm{P}-\mathrm{C} 1 \mathrm{P}-\mathrm{N} 1 \mathrm{P}$ & $126(4)$ \\
\hline $\mathrm{C} 15-\mathrm{C} 14-\mathrm{C} 13$ & $119.3(6)$ & $\mathrm{O} 1 \mathrm{P}-\mathrm{C} 1 \mathrm{P}-\mathrm{C} 2 \mathrm{P}$ & $128(4)$ \\
\hline $\mathrm{C} 15-\mathrm{C} 14-\mathrm{H} 14$ & 120.4 & $\mathrm{~N} 1 \mathrm{P}-\mathrm{C} 1 \mathrm{P}-\mathrm{C} 2 \mathrm{P}$ & $106(3)$ \\
\hline $\mathrm{C} 13-\mathrm{C} 14-\mathrm{H} 14$ & 120.4 & $\mathrm{C} 3 \mathrm{P}-\mathrm{C} 2 \mathrm{P}-\mathrm{C} 1 \mathrm{P}$ & $104(3)$ \\
\hline $\mathrm{C} 14-\mathrm{C} 15-\mathrm{C} 10^{\mathrm{iv}}$ & $121.0(6)$ & $\mathrm{C} 3 \mathrm{P}-\mathrm{C} 2 \mathrm{P}-\mathrm{H} 2 \mathrm{P} 1$ & 110.9 \\
\hline $\mathrm{C} 14-\mathrm{C} 15-\mathrm{C} 18^{\mathrm{v}}$ & $119.6(6)$ & $\mathrm{C} 1 \mathrm{P}-\mathrm{C} 2 \mathrm{P}-\mathrm{H} 2 \mathrm{P} 1$ & 110.9 \\
\hline $\mathrm{C} 10^{\mathrm{iv}}-\mathrm{C} 15-\mathrm{C} 18^{\mathrm{v}}$ & $119.4(6)$ & $\mathrm{C} 3 \mathrm{P}-\mathrm{C} 2 \mathrm{P}-\mathrm{H} 2 \mathrm{P} 2$ & 111.0 \\
\hline $\mathrm{O} 8-\mathrm{C} 16-\mathrm{O} 7$ & $124.3(7)$ & $\mathrm{C} 1 \mathrm{P}-\mathrm{C} 2 \mathrm{P}-\mathrm{H} 2 \mathrm{P} 2$ & 111.0 \\
\hline
\end{tabular}




$\begin{array}{llll}\mathrm{O} 8-\mathrm{C} 16-\mathrm{C}^{\mathrm{vi}} & 120.8(7) & \mathrm{H} 2 \mathrm{P} 1-\mathrm{C} 2 \mathrm{P}-\mathrm{H} 2 \mathrm{P} 2 & 109.0 \\ \mathrm{O} 7-\mathrm{C} 16-\mathrm{C}^{\mathrm{vi}} & 114.9(6) & \mathrm{C} 4 \mathrm{P}-\mathrm{C} 3 \mathrm{P}-\mathrm{C} 2 \mathrm{P} & 105(3) \\ \mathrm{O} 10-\mathrm{C} 17-\mathrm{O} 9 & 124.3(6) & \mathrm{C} 4 \mathrm{P}-\mathrm{C} 3 \mathrm{P}-\mathrm{H} 3 \mathrm{P} 1 & 110.7 \\ \mathrm{O} 10-\mathrm{C} 17-\mathrm{C}^{\mathrm{v}} & 121.3(6) & \mathrm{C} 2 \mathrm{P}-\mathrm{C} 3 \mathrm{P}-\mathrm{H} 3 \mathrm{P} 1 & 110.7 \\ \mathrm{O} 9-\mathrm{C} 17-\mathrm{C}^{\mathrm{v}} & 114.4(6) & \mathrm{C} 4 \mathrm{P}-\mathrm{C} 3 \mathrm{P}-\mathrm{H} 3 \mathrm{P} 2 & 110.7 \\ \mathrm{O} 12-\mathrm{C} 18-\mathrm{O} 11 & 125.5(6) & \mathrm{C} 2 \mathrm{P}-\mathrm{C} 3 \mathrm{P}-\mathrm{H} 3 \mathrm{P} 2 & 110.7 \\ \mathrm{O} 12-\mathrm{C} 18-\mathrm{C} 15^{\mathrm{i}} & 120.2(6) & \mathrm{H} 3 \mathrm{P} 1-\mathrm{C} 3 \mathrm{P}-\mathrm{H} 3 \mathrm{P} 2 & 108.8 \\ \mathrm{O} 11-\mathrm{C} 18-\mathrm{C} 15^{\mathrm{i}} & 114.2(6) & \mathrm{C} 3 \mathrm{P}-\mathrm{C} 4 \mathrm{P}-\mathrm{N} 1 \mathrm{P} & 102(3) \\ \mathrm{O} 13-\mathrm{C} 19-\mathrm{N} 1 & 124.4(6) & \mathrm{C} 3 \mathrm{P}-\mathrm{C} 4 \mathrm{P}-\mathrm{H} 4 \mathrm{P} 1 & 111.3 \\ \mathrm{O} 13-\mathrm{C} 19-\mathrm{C} 20 & 126.3(6) & \mathrm{N} 1 \mathrm{P}-\mathrm{C} 4 \mathrm{P}-\mathrm{H} 4 \mathrm{P} 1 & 111.3 \\ \mathrm{~N} 1-\mathrm{C} 19-\mathrm{C} 20 & 109.3(6) & \mathrm{C} 3 \mathrm{P}-\mathrm{C} 4 \mathrm{P}-\mathrm{H} 4 \mathrm{P} 2 & 111.3 \\ \mathrm{C} 19-\mathrm{C} 20-\mathrm{C} 21 & 104.2(6) & \mathrm{N} 1 \mathrm{P}-\mathrm{C} 4 \mathrm{P}-\mathrm{H} 4 \mathrm{P} 2 & 111.3 \\ \mathrm{C} 19-\mathrm{C} 20-\mathrm{H} 20 \mathrm{~A} & 110.9 & \mathrm{H} 4 \mathrm{P} 1-\mathrm{C} 4 \mathrm{P}-\mathrm{H} 4 \mathrm{P} 2 & 109.2 \\ \mathrm{C} 21-\mathrm{C} 20-\mathrm{H} 20 \mathrm{~A} & 110.9 & \mathrm{~N} 1 \mathrm{P}-\mathrm{C} 5 \mathrm{P}-\mathrm{H} 5 \mathrm{P} 1 & 109.5 \\ \mathrm{C} 19-\mathrm{C} 20-\mathrm{H} 20 \mathrm{~B} & 110.9 & \mathrm{~N} 1 \mathrm{P}-\mathrm{C} 5 \mathrm{P}-\mathrm{H} 5 \mathrm{P} 2 & 109.5 \\ \mathrm{C} 21-\mathrm{C} 20-\mathrm{H} 20 \mathrm{~B} & 110.9 & \mathrm{H} 5 \mathrm{P} 1-\mathrm{C} 5 \mathrm{P}-\mathrm{H} 5 \mathrm{P} 2 & 109.5 \\ \mathrm{H} 20 \mathrm{~A}-\mathrm{C} 20-\mathrm{H} 20 \mathrm{~B} & 108.9 & \mathrm{~N} 1 \mathrm{P}-\mathrm{C} 5 \mathrm{P}-\mathrm{H} 5 \mathrm{P} 3 & 109.5 \\ \mathrm{C} 22-\mathrm{C} 21-\mathrm{C} 20 & 105.2(6) & \mathrm{H} 5 \mathrm{P} 2-\mathrm{C} 5 \mathrm{P}-\mathrm{H}-\mathrm{H} 35 & 109.5 \\ \mathrm{C} 22-\mathrm{C} 21-\mathrm{H} 21 \mathrm{~A} & 110.7 & \mathrm{C} 1 \mathrm{P}-\mathrm{N} 1 \mathrm{P}-\mathrm{C} 5 \mathrm{P} & 122(4) \\ \mathrm{C} 20-\mathrm{C} 21-\mathrm{H} 21 \mathrm{~A} & 110.7 & \mathrm{C} 1 \mathrm{P}-\mathrm{N} 1 \mathrm{P}-\mathrm{C} 4 \mathrm{P} & 116(3) \\ \mathrm{C} 22-\mathrm{C} 21-\mathrm{H} 21 \mathrm{~B} & 110.7 & \mathrm{C} 5 \mathrm{P}-\mathrm{N} 1 \mathrm{P}-\mathrm{C} 4 \mathrm{P} & 122(4) \\ \mathrm{C} 20-\mathrm{C} 21-\mathrm{H} 21 \mathrm{~B} & 110.7 & & \end{array}$

Symmetry codes: (i) $x+1, y, z$; (ii) $x+1 / 2,-y+2, z+1 / 2$; (iii) $x+1 / 2,-y+1, z+1 / 2$; (iv) $x-1 / 2,-y+1, z-1 / 2$; (v) $x-1, y, z$; (vi) $x-1 / 2,-y+2, z-1 / 2$.

Hydrogen-bond geometry $\left(A,{ }^{\circ}\right)$

\begin{tabular}{lllll}
\hline$D-\mathrm{H} \cdots A$ & $D-\mathrm{H}$ & $\mathrm{H} \cdots A$ & $D \cdots A$ & $D-\mathrm{H} \cdots A$ \\
\hline $\mathrm{N} 3-\mathrm{H} 8 N \cdots \mathrm{O} 1 P$ & $0.89(3)$ & $1.60(7)$ & $2.47(6)$ & $167(7)$ \\
$\mathrm{N} 3-\mathrm{H} 8 N \cdots \mathrm{O} 1 S$ & $0.89(3)$ & $1.91(3)$ & $2.779(9)$ & $166(6)$ \\
$\mathrm{N} 3-\mathrm{H} 7 N \cdots \mathrm{O} 12^{\text {vii }}$ & $0.88(3)$ & $1.97(4)$ & $2.786(6)$ & $154(6)$ \\
$\mathrm{N} 3-\mathrm{H} 6 N \cdots \mathrm{O} 9$ & $0.87(3)$ & $2.03(3)$ & $2.867(7)$ & $161(6)$ \\
$\mathrm{N} 3-\mathrm{H} 5 N \cdots \mathrm{O} 4^{\mathrm{v}}$ & $0.86(3)$ & $1.94(3)$ & $2.800(7)$ & $174(6)$ \\
$\mathrm{N} 2-\mathrm{H} 4 N \cdots \mathrm{O} 13^{\text {viii }}$ & $0.86(3)$ & $1.85(3)$ & $2.713(7)$ & $173(6)$ \\
$\mathrm{N} 2-\mathrm{H} 3 N \cdots \mathrm{O} 11^{\text {vii }}$ & $0.88(3)$ & $2.24(4)$ & $3.025(7)$ & $148(6)$ \\
$\mathrm{N} 2-\mathrm{H} 3 N \cdots \mathrm{O} 1^{\text {vii }}$ & $0.88(3)$ & $2.41(5)$ & $3.104(7)$ & $136(6)$ \\
$\mathrm{N} 2-\mathrm{H} 2 N \cdots \mathrm{O} 8^{\text {iii }}$ & $0.88(3)$ & $1.91(4)$ & $2.737(7)$ & $156(6)$ \\
$\mathrm{N} 2-\mathrm{H} 1 N \cdots \mathrm{O} 10^{\text {ix }}$ & $0.88(3)$ & $1.97(3)$ & $2.825(7)$ & $163(6)$
\end{tabular}

Symmetry codes: (iii) $x+1 / 2,-y+1, z+1 / 2$; (v) $x-1, y, z$; (vii) $x-1 / 2,-y+1, z+1 / 2$; (viii) $x+1 / 2,-y, z+1 / 2$; (ix) $x, y-1, z$. 